\title{
Adaptive Diagnosis for Rotating Machineries Using Information Geometrical Kernel-ELM Based on VMD-SVD
}

\author{
Zhipeng Wang ${ }^{1,2,3, *(1)}$, Limin Jia ${ }^{1,2,3}$ and Yong Qin $1,2,3$ \\ 1 State Key Lab of Rail Traffic Control and Safety, Beijing Jiaotong University, Beijing 100044, China; \\ lmjia@bjtu.edu.cn (L.J.); yqin@bjtu.edu.cn (Y.Q.) \\ 2 National Engineering Laboratory for System Safety and Operation Assurance of Urban Rail Transit, \\ Guangzhou 510000, China \\ 3 Beijing Research Center of Urban Traffic Information Sensing and Service Technologies, \\ Beijing Jiaotong University, Beijing 100044, China \\ * Correspondence: zpwang@bjtu.edu.cn; Tel.: +86-010-5168-4281
}

Received: 6 December 2017; Accepted: 16 January 2018; Published: 21 January 2018

\begin{abstract}
Rotating machineries often work under severe and variable operation conditions, which brings challenges to fault diagnosis. To deal with this challenge, this paper discusses the concept of adaptive diagnosis, which means to diagnose faults under variable operation conditions with self-adaptively and little prior knowledge or human intervention. To this end, a novel algorithm is proposed, information geometrical extreme learning machine with kernel (IG-KELM). From the perspective of information geometry, the structure and Riemannian metric of Kernel-ELM is specified. Based on the geometrical structure, an IG-based conformal transformation is created to improve the generalization ability and self-adaptability of KELM. The proposed IG-KELM, in conjunction with variation mode decomposition (VMD) and singular value decomposition (SVD) is utilized for adaptive diagnosis: (1) VMD, as a new self-adaptive signal processing algorithm is used to decompose the raw signals into several intrinsic mode functions (IMFs). (2) SVD is used to extract the intrinsic characteristics from the matrix constructed with IMFs. (3) IG-KELM is used to diagnose faults under variable conditions self-adaptively with no requirement of prior knowledge or human intervention. Finally, the proposed method was applied on fault diagnosis of a bearing and hydraulic pump. The results show that the proposed method outperforms the conventional method by up to $7.25 \%$ and $7.78 \%$ respectively, in percentages of accuracy.
\end{abstract}

Keywords: fault diagnosis; information geometry; kernel extreme learning machine; variation mode decomposition

\section{Introduction}

As industrial systems become more and more sophisticated, slight faults may result in catastrophes. Therefore, fault diagnosis technology has become more and more significant for the safety and reliable utilization of systems [1]. As we known, failures of rotating machineries are common causes of breakdown in industries. The growing demand for safety and reliability in industries requires a smart fault diagnosis system for rotating machineries [2]. Many researchers have studied the implementations of fault diagnosis algorithms on mobile devices for wider adoption [3-6]. Not only can the efficient fault diagnosis keep the monitored systems healthy and safe, they can also decrease the cost of repairs or replacements [7].

A lot of research have been done on the fault diagnosis for rotating machineries during the past decades. Vibration analysis is the main method for condition monitoring of rotating machineries [8]. 
Currently, a lot of data-driven methods have been proposed, for example, fuzzy logic $[2,8]$, principal component analysis $[9,10]$. Most of the methods mainly require certain and stable operation conditions. However, it is known that real-world machineries are usually operating under variable and uncertain conditions. Sometimes, the undergoing operation condition is even unknown. It is quite challenging that a diagnosis model trained under condition $\mathrm{A}$ is used under another unknown condition $\mathrm{B}$. Misdiagnosis will emerge considerably in this situation.

Many studies have been done on this subject. Yu et al. [10] proposed a hybrid feature selection method to deal with it, but required prior knowledge about the undergoing conditions. Liu et al. [11] and Tian et al. [12] employed multifractal detrended fluctuation analysis and permutation entropy to diagnose bearing faults under fluctuations in operation conditions, but required human intervention for the final clustering. Tian et al. [13] employed extreme learning machine for bearing fault diagnosis under variable conditions, but required training data under all conditions. However, it is even impossible to acquire such prior knowledge and training data under all possible operation conditions. That is because the real undergoing operation conditions are often unknown and uncertain. Therefore, it is valuable to develop a new fault diagnosis method which can adapt itself to unknown variable conditions with little prior knowledge or human intervention. In this paper, it is described as "adaptive diagnosis".

An adaptive diagnosis method should be provided with two characteristics:

(1) Adaptivity to unknown variable conditions. Rotating machineries often work under unknown variable conditions without any prior knowledge or training data. Since the health characteristics of rotating machineries might be variable under different conditions, an adaptive diagnosis method ought to be self-adaptive to unknown variable conditions.

(2) Automaticity with little human intervention. An adaptive diagnosis method should be applied automatically and independent of human intervention as much as possible.

Generally, if a fault diagnosis method satisfies the above characteristics, it can be considered as an adaptive diagnosis method. The application of adaptive diagnosis methods can not only reduce the dependence on operators' experiences and skills, but also decrease the complexity and cost of condition monitoring systems. This paper attempts to propose an adaptive diagnosis method for rotating machineries by using vibration analysis.

Feature extraction is the basic step during fault diagnosis. It should be self-adaptive and provides remarkable features with little human intervention to extract valid features under variable conditions. Currently, a lot time-frequency analysis methods have been employed for feature extraction for rotating machineries, such as bearing, gearbox [14-22]. Wavelet transform, as a well-known time-frequency analysis tool, has been employed wildly to decompose the nonstationary signals. However, it still suffers several unacceptable disadvantages [23-25]. It is considered as an improved Fourier transform with adjustable windows [26]. The structure of the wavelet basic function is permanent during the decomposition $[23,27]$. Obviously, its non-adaptive nature is not appropriate for adaptive diagnosis.

Empirical mode decomposition (EMD), proposed by Huang et al. [28] is another well-known method and can decompose signals to several intrinsic mode functions (IMFs) according to the time and scale characteristics. It is completely self-adaptive and seems suitable for adaptive diagnosis [28-32]. However, is suffers from many problems, such as: modal aliasing, pseudo components, end effect and so on. Therefore, a more efficient self-adaptive method is needed.

Variation mode decomposition (VMD), as a novel self-adaptive method, was proposed by Dragomiretskiy et al. in 2014 [33]. It is a non-recursive method to decompose signals into quasi-orthogonal IMFs, each with a center frequency. It has been proved to overcome the problems of EMD and its extended methods [34]. Therefore, VMD is employed for feature extraction.

The IMFs acquired by VMD can be used to form a matrix, which is too large to be directly used for fault diagnosis. Therefore, a suitable algorithm is needed to extract the intrinsic characteristics of the matrix. Singular value decomposition (SVD), which has been proven to extract the features to periodic impulses is employed to obtain intrinsic features from the matrix with favorable stability $[35,36]$. 
After feature extraction, the next step is fault clustering. As aforementioned, the fault clustering method should adapt to unknown variable conditions automatically with little prior knowledge or human intervention. Therefore, the traditional discriminant analysis methods, such as Mahalanobis distance [37-39] and Fisher discriminant analysis [40,41], are not suitable, because they mainly require certain levels of expertise and threshold settings. In contrast, computation intelligence techniques are preferred in this situation [42]. The computation intelligence techniques are data-driven and have been employed broadly in fault diagnosis, such as: Bayes net classifier [43], optimization algorithms [44,45] and artificial neural networks [46-48]. Deep learnings, such as efficient and remarkable algorithms have been employed automatically with little human intervention [49-51]. They are capable to even extract feature self-adaptively and seems suitable for adaptive diagnosis. Deep learnings are data-hungry and require plenty of training data which are hardly acquired in practice, especially the faulty data under different conditions.

Extreme learning machine (ELM), proposed by Huang [52,53], has been proven as an efficient algorithm for regression and multi-classification [54]. Compared with the conventional gradient-based algorithms and support vector machine (SVM), ELM spends less running time and performs better [55]. On this basis, Kernel-ELM (KELM) [56] is proposed by using a kernel function to improve the generalization ability and reduce possible over-fitting problems.

Similar to SVM, the performance of KELM also relies on the kernel function. But the choosing of the kernel function mainly depends on prior knowledge and expertise [55-57]. However, different kernel types or parameters maybe only fit several specific datasets respectively. Whereas measured signals under different conditions may prefer different kernel types or parameters. Therefore, it is necessary to modify the algorithm self-adaptively, to make sure that the diagnosis method is insensitive enough to the manual configuration of kernel and performs acceptably even if the kernel types or parameters are set badly.

Information geometry (IG), proposed by Amari [58], which aims to analyze information theory, statistics and machine learning based on differential geometry, offers a feasible approach. It can analyze and modify machine learning algorithms by convex analysis and constructing differential manifolds. By elucidating the dualistic differential-geometrical structure, information geometry has been widely applied [59-64].

In this paper, motivated by information geometry, we propose a novel algorithm, information geometrical kernel-ELM (IG-KELM). Firstly, we specify the geometrical structure and Riemannian metric of Kernel-ELM from the perspective of information geometry. Then, a data-dependent conformal transformation is created with Mahalanobis distance to modify the KELM self-adaptively. IG-KELM is insensitive to inappropriate kernel configuration, and can adapt itself to signals under variable conditions with little prior knowledge or human intervention. The feasibility and effectivity of IG-KELM was verified by simulation experiments.

The outline of this paper is as follows: Section 2 introduces VMD, Kernel-ELM, Riemannian metric of Kernel-ELM, information geometrical kernel-ELM, as well as the scheme of the proposed method; Section 3 describes the simulation experiment performed to verify IG-KELM; Section 4 describes the applications of the proposed method on fault diagnosis for bearing and hydraulic pump; and Section 5 is the conclusions.

\section{Methodology}

As aforementioned, VMD is the core algorithm for feature extraction, and kernel-ELM is the basic algorithm for fault clustering. In this section, VMD and kernel-ELM is introduced firstly. Then, the analysis of Riemannian metric of Kernel-ELM is described. On this basis, the IG-KELM is proposed. At the end of this section, the scheme of adaptive diagnosis based on VMD-SVD and IG-KELM is described. 


\subsection{Variational Mode Decomposition}

VMD is capable to estimate the modes and determine the correlative bands of fault feature at the same time, which can decompose the signal into several IMFs [33]. VMD is a constrained variational problem represented by the following equation:

$$
\begin{gathered}
\min _{\mu_{k}, \omega_{k}}\left\{\sum_{k}\left\|\partial_{t}\left[\left(\sigma_{t}+\frac{j}{\pi t}\right) \mu_{k}(t)\right] e^{-j t \omega_{k}}\right\|_{2}^{2}\right\}, \\
\text { subject to } \quad \sum_{k} \mu_{k}=f
\end{gathered}
$$

where $\mu_{k}$ and $\omega_{k}$ are IMF components and their center frequencies.

To obtain the optimal solution of the problem, introduce the augmented Lagrange function:

$$
L\left(\mu_{k}, \omega_{k}, \lambda\right)=\alpha \sum_{k}\left\|\partial_{t}\left[\left(\sigma_{t}+\frac{j}{\pi t}\right) \mu_{k}(t)\right] e^{-j t \omega_{k}}\right\|_{2}^{2}+\left\|f-\sum \mu_{k}\right\|_{2}^{2}+\left\langle\lambda, f-\sum \mu_{k}\right\rangle .
$$

The mode number $k$ and quadratic penalty $\alpha$ are set in advance, while the sub-mode function $\mu_{k}{ }^{1}$, the center frequency $\omega_{k}^{1}$ and the Largrangian multiplier $\lambda^{1}$ are initialized [33]. Then modes $\mu_{k}$ and the center frequency $\omega_{k}$ are renewed respectively by Equations (3) and (4):

$$
\begin{gathered}
\mu_{k}^{n+1} \leftarrow \frac{\hat{f}-\sum_{i<k} \hat{\mu}_{i}^{n+1}-\sum_{i>k} \hat{\mu}_{i}^{n}+\frac{\hat{\lambda}^{n}}{2}}{1+2 \alpha\left(\omega-\omega_{k}^{n}\right)^{2}}, k \in\{1, K\}, \\
\omega_{k}^{n+1} \leftarrow \frac{\int_{0}^{\infty} \omega\left|\widehat{\mu}_{k}^{n+1}(\omega)\right|^{2} d \omega}{\int_{0}^{\infty}\left|\widehat{\mu}_{k}^{n+1}(\omega)\right|^{2} d \omega}, k \in\{1, K\} .
\end{gathered}
$$

After the modes and center frequencies are updated, the Largrangian multiplier $\lambda$ is also updated by Equation (5):

$$
\lambda^{n+1}=\lambda^{n}+\tau\left(x-\sum_{k} \mu_{k}^{n+1}\right)
$$

$\mu_{k}, \omega_{k}$ and $\lambda$ are updated iteratively until Equation (6) is satisfied.

$$
\sum_{k}\left\|\mu_{k}^{n+1}-\mu_{k}^{n}\right\|_{2}^{2} /\left\|\mu_{k}^{n}\right\|_{2}^{2}<\varepsilon
$$

\subsection{Kernel-ELM}

ELM is originally developed for the training of Single hidden layer feedforward networks (SLFNs) and then extended to the generalized SLFNs. The architecture of ELM is shown in Figure 1. The details about ELM can be found in Huang et al. [52,53]. Consider a dataset $\left(x_{i}, y_{i}\right)$, where $x_{i}(i=1,2, \cdots, N)$ is input vector, $y_{i}(i=1,2, \cdots, M)$ is output vector. The output function of ELM can be described as:

$$
f_{L}(x)=\sum_{i=1}^{L} \beta_{i} G\left(\alpha_{i}, b_{i}, x\right)=h(x) \cdot \beta
$$

where, $L$ is the number of hidden neurons, $\alpha_{i}$ is the weight vector connecting the $i$ th hidden node and the input nodes, $b_{i}$ is the bias of the $i$ th hidden node, $\beta_{i}$ is the weight vector connecting the $i$ th hidden node and the output nodes, $G(\bullet)$ represents the hidden nodes activation function. $h(x)$ is the hidden layer output matrix of the network. 


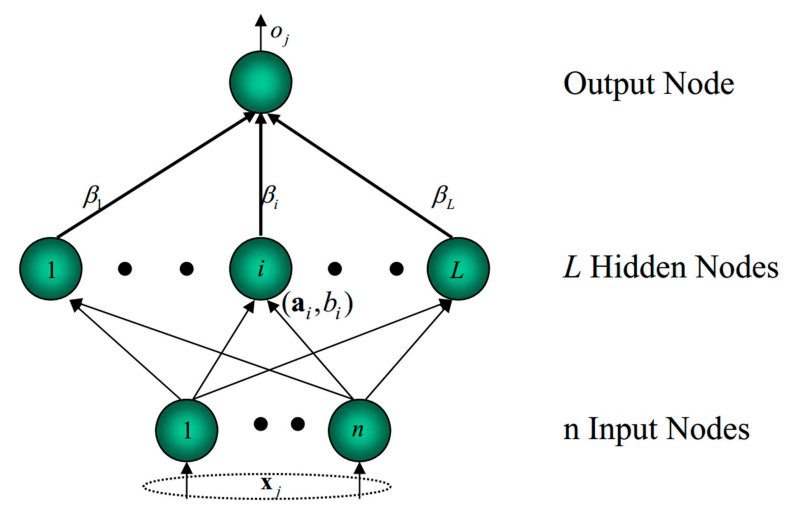

Figure 1. The architecture of ELM.

According to the ELM, $\alpha_{i}$ and $b_{i}$ are randomly assigned and the least square solution of $\beta$ is computed by the following objective function:

$$
\left\{\begin{array}{c}
\min \sum_{i=1}^{N}\left\|\beta \cdot h\left(x_{i}\right)-y_{i}\right\| \\
\min \|\beta\|
\end{array} .\right.
$$

Therefore,

$$
\widehat{\beta}=H^{+} Y,
$$

where, $\mathrm{H}^{+}$is the Moore-Penrose pseudo inverse of matrix $H$. Details can be found in Huang et al. [53].

To improve the generalization ability and reduce possible over-fitting problems, the training error is not supposed to be equal to zero. Therefore, the objective function can be rewritten as:

$$
\left\{\begin{array}{c}
\min \sum_{i=1}^{N} \frac{1}{2}\|\beta\|^{2}+C \sum_{i=1}^{N} \xi_{i} \\
\text { subject to } h\left(x_{i}\right) \beta=y_{i}^{T}-\xi_{i}^{T} \quad i=1,2, \cdots
\end{array},\right.
$$

where, $\xi_{i}$ is the training error of the $i$ th input vector, $C$ is the regularization parameter. The optimal value of $\beta$ can be obtained as:

$$
\widehat{\beta}=H^{T}\left(\frac{I}{C}+H H^{T}\right)^{-1} Y .
$$

If $h(x)$ is unknown, the Mercer's conditions are introduced to the ELM model, a kernel function is defined as:

$$
K\left(x_{i}, x_{j}\right)=<h\left(x_{i}\right), h\left(x_{j}\right)>.
$$

Therefore, the output function can be rewritten as:

$$
f_{L}(x)=h(x) \cdot \beta=h(x) H^{T}\left(\frac{I}{C}+H H^{T}\right)^{-1} Y=\left[\begin{array}{c}
K\left(x, x_{j}\right) \\
\vdots \\
K\left(x, x_{N}\right)
\end{array}\right]^{T}\left(\frac{I}{C}+\sum_{i, j=1}^{N} K\left(x_{i}, x_{j}\right)\right)^{-1} Y .
$$

The regularization parameter $C$ is usually calculated by using $n$-fold cross-validation (CV) method [56]. From the Equation (13), it is shown that the output function is determined by the kernel function. There are several popular kernel functions, such as: polynomial functions, radial basis functions and so on. Different kernel functions are appropriate for different situations. 


\subsection{Riemannian Metric of Kernel-ELM}

For classification, KELM supposes to find an optimal separating hyperplane, which passes through the origin of the KELM random feature space [55]. To modify the kernel function data-dependently, information geometry is employed to analyze the structure of kernel mapping geometrically. According to information geometry [59], $h(x)$ is considered as an embedding of the input space $S$ into the random feature space $F$ as a curved submanifold. The mapped pattern of $x$ is $z: z=h(x)$. It can be expressed in differential form:

$$
d z=\nabla h(x) \cdot d x=\sum_{i} \frac{\partial}{\partial x_{i}} h(x) \cdot d x_{i}
$$

here, the squared length of $d z=\left(d z_{\alpha}\right)$ can be described as:

$$
|d z|=\sum_{\alpha}\left(d z_{\alpha}\right)^{2}=\sum_{i, j} g_{i j}(x) d x_{i} d x_{j}
$$

where:

$$
g_{i j}(x)=\left(\frac{\partial}{\partial x_{i}} h(x)\right) \cdot\left(\frac{\partial}{\partial x_{j}} h(x)\right) .
$$

$G(x)=\left(g_{i j}(x)\right)$ is the Riemannian metric tensor in the input space. It shows that $G(x)$ is a positive-definite matrix. According to the theorems introduced by Wu et al. [65], $g_{i j}(x)$ can be described as:

$$
g_{i j}(x)=\left.\frac{\partial}{\partial x_{i}} \frac{\partial}{\partial x^{\prime}} K\left(x, x^{\prime}\right)\right|_{x^{\prime}=x}=\left(\frac{1}{2} \frac{\partial^{2} K(x, x)}{\partial x_{i} \partial x_{j}}-\frac{\partial^{2} K\left(x, x^{\prime}\right)}{\partial x_{i} \partial x_{j}}\right)_{x^{\prime}=x} .
$$

It is clear that the Riemannian metric $G(x)$ can be directly determined by the kernel. Therefore, for Polynomial kernel, the induced Riemannian metric is:

$$
g_{i j}(x)=d \delta_{i j}+x_{i} x_{j} d(d-1)
$$

For Gaussian radial basis function, the induced Riemannian metric is:

$$
g_{i j}(x)=2 \gamma \cdot \delta_{i j}
$$

where:

$$
\delta_{i j}=\left\{\begin{array}{ll}
1 & i=j \\
0 & i \neq j
\end{array} .\right.
$$

Therefore, the structure of kernel-ELM can be analyzed geometrically by using the Riemannian metric.

\subsection{Information Geometrical Kernel-ELM}

To modify KELM self-adaptively, we have to improve the generalization ability of KELM by using data rather than prior knowledge or expertise. From the perspective of the geometry, it can be found easily that the improvement of the spatial resolution around the optimal hyperplane will enhance the separability of patterns. In this study, a conformal transformation is utilized:

$$
\widehat{K}\left(x, x^{\prime}\right)=D(x) D\left(x^{\prime}\right) K\left(x, x^{\prime}\right),
$$

where $\widehat{K}\left(x, x^{\prime}\right)$ is the modified kernel function and $D(x)$ is a positive scalar function. The new Riemannian metric $\widehat{g}_{i j}(x)$ can be rewritten as:

$$
\widehat{g}_{i j}(x)=D(x)^{2} g_{i j}(x)+D_{i}(x) D_{j}(x)+2 D_{i}(x) D(x) K(x, x),
$$


where:

$$
\left\{\begin{array}{c}
D_{i}(x)=\partial D(x) / \partial x_{i} \\
K_{i}(x, x)=\partial K\left(x, x^{\prime}\right) /\left.\partial x_{i}\right|_{x^{\prime}=x}
\end{array} .\right.
$$

The selection of the factor $D(x)$ should follow the rule that its value is greater when $x$ is approaching to the boundary, and smaller when $x$ is further away from the boundary. Therefore, the spatial resolution around the optimal hyperplane is enhanced.

However, the position of the hyperplane is unknown in practice. To solve this problem, we utilize Mahalanobis distance (MD) to estimate the approximate position of the hyperplane. The MD can be described as:

$$
M D_{i}^{(k)}=\left(x_{i}-u^{k}\right)^{T} \sum^{-1}\left(x_{i}-u^{k}\right),
$$

where, $M D_{i}^{(k)}$ represents the distance between $x_{i}$ and the $k$ th pattern, $u^{k}$ and $\Sigma^{-1}$ represents the mean vector and covariance matrix of the $k$ th pattern.

In view of the aforementioned analysis, the conformal mapping $D(x)$ is given as:

$$
D\left(x_{i}\right)=\frac{1}{M} \sum_{k=1}^{M} \exp \left(-\frac{1}{M D_{i}^{(k)}}\right),
$$

where, $M$ is the number of patterns. It is shown that the chosen $D(x)$ is directly derived from the data only and follows the aforementioned rule. By using this approach, this study improves the generalization ability of KELM self-adaptively based on the training data with no requirement of prior knowledge or expertise.

In general, a novel algorithm called information geometrical Kernel-ELM (IG-KELM) is proposed, as shown in Figure 2:

1. Train the KELM with a primary kernel $K$;

2. Calculate Mahalanobis distances to obtain the conformal mapping $D(x)$;

3. Transform the kernel $K$ by Equation (21), and obtain the modified kernel $\widehat{K}$;

4. Retrain the KELM with the new kernel $\widehat{K}$.

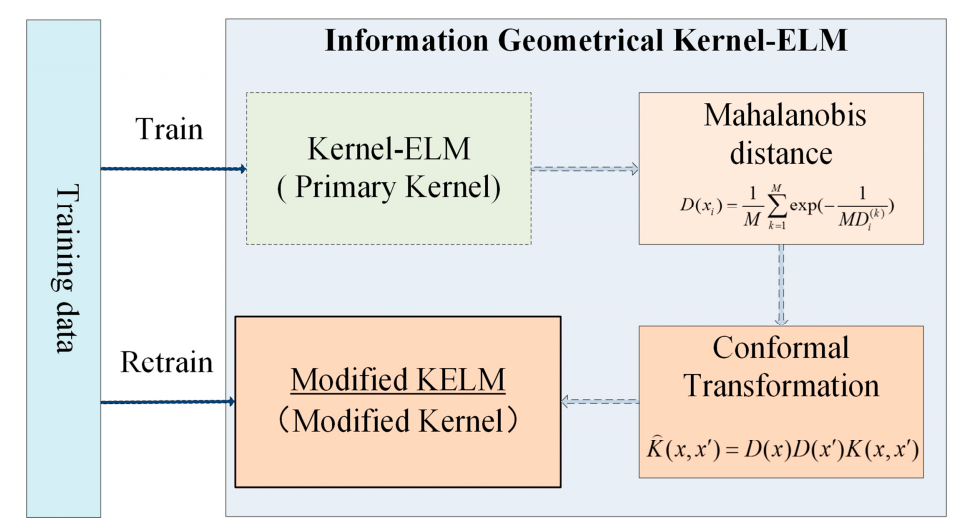

Figure 2. The scheme of IG-KELM.

\subsection{Adaptive Diagnosis Based on VMD-SVD and IG-KELM}

In this study, self-adaptive algorithms are used for both feature extraction and fault clustering; VMD-SVD is used for feature extraction and IG-KELM is used for fault clustering.

1. Feature extraction. VMD decomposes each signal into $n$-empirical modes (IMFs) self-adaptively, and the IMFs are constructed as a matrix. Then, SVD is employed to get a $n$-dimensional feature vector of singular values from the matrix. 
2. Fault clustering. The proposed IG-KELM is employed for fault diagnosis under variable conditions.

The proposed method is self-adaptive and has strong robustness. Those advantages imply that this method is able to diagnose fault under unknown operation conditions without corresponding training data. The proposed method can be implemented self-adaptively under variable conditions, with little requirement of prior knowledge, expertise, parameter configuration or any other human intervention.

\section{Simulation Experiment for IG-KELM}

A comparison of simulation experiments between IG-KELM and the conventional KELM was made to verify the efficiency and self-adaptivity while using different kernel types and parameters.

\subsection{Simulation Data}

Suppose that there is a simulated two-dimensional dataset $Z=(x, y)$ which is distributed evenly in the region $[-2,2] \times[-2,2]$. The datasets are divided into two classes using a curve determined by $y=\sin (x)+\frac{\sin (3 x)}{3}-2 \sin \left(\frac{x}{2}\right)$. Then, the IG-KELM or KELM produces a new boundary to cluster the dataset. The accuracy of the classification can be employed to verify the efficiency and self-adaptivity of IG-KELM or KELM while using different kernel functions and parameters.

In this simulation, 500 training samples were randomly and uniformly generated, as shown in Figure 3. Another 2000 test samples were generated for verification. Two types of kernel functions were involved, Gaussian RBF and Polynomial kernels in this study. In IG-KELM.

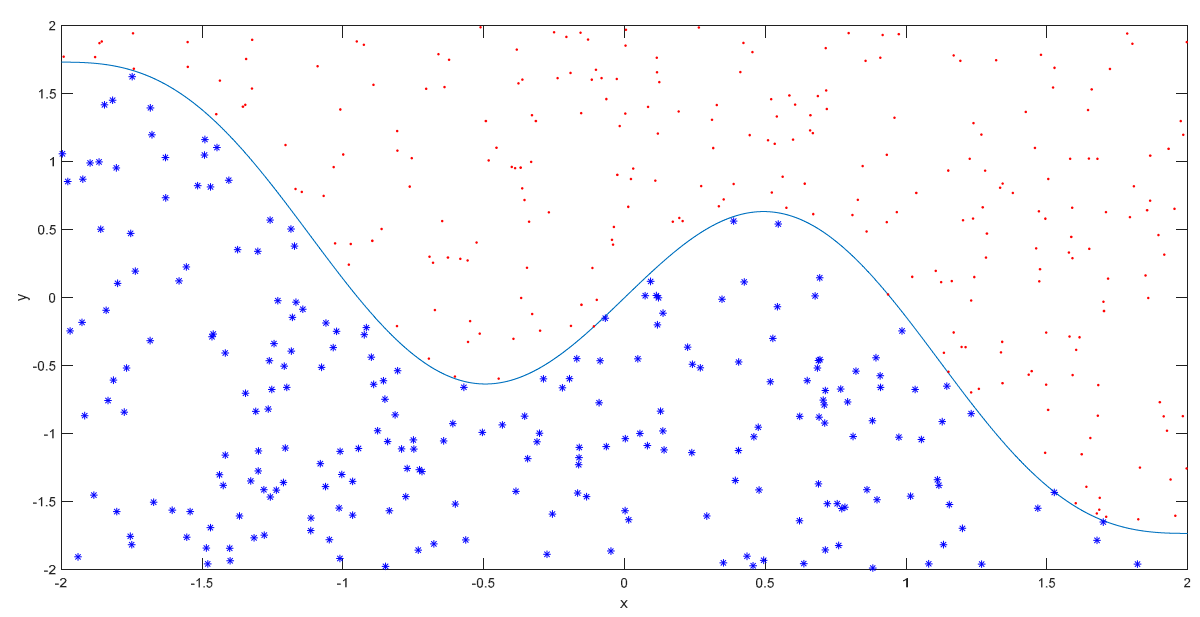

Figure 3. 500 training samples of simulation experiment.

\subsection{Simulation Results}

\subsubsection{By Using the Gaussian RBF Kernel}

As aforementioned, 500 training data were used to train the KELM with a Gaussian RBF kernel. According to Huang et al. [56], the regularization parameter $C$ can be selected from $\left[2^{-20}, 2^{-19}\right.$, $\left.\ldots, 2^{19}, 2^{20}\right]$, and kernel parameter $\gamma$ can be selected from $\left[2^{-10}, 2^{-9}, \ldots, 2^{9}, 2^{10}\right]$. A five-fold cross-validation (CV) method is employed to optimize the parameters, as shown in Figure 4. As a result, the optimal $C$ and $\gamma$ were $2^{5}$ and $2^{-3}$ respectively. 


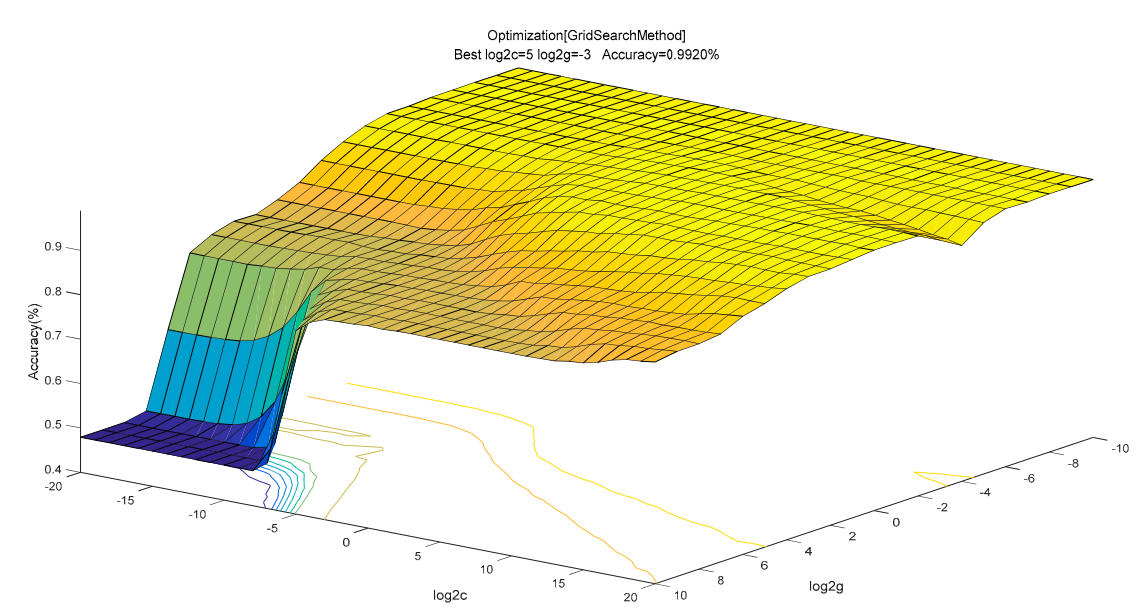

Figure 4. Optimization of parameters by using 5-fold cross-validation (CV) method.

Then, the classification results of KELM and IG-KELM were calculated, as shown in Table 1. The results show that the test accuracy of KELM is $99.20 \%$, while the test accuracy of IG-KELM is $99.65 \%$. Therefore, the trained IG-KELM performed better than KELM.

Table 1. Simulation experiments of KELM and IG-KELM with an RBF kernel.

\begin{tabular}{ccccccc}
\hline & \multicolumn{3}{c}{ KELM } & \multicolumn{3}{c}{ IG-KELM } \\
\cline { 2 - 6 } & $\begin{array}{c}\text { Training } \\
\text { Error Rate }\end{array}$ & $\begin{array}{c}\text { Test } \\
\text { Error Rate }\end{array}$ & $\begin{array}{c}\text { Test } \\
\text { Accuracy }\end{array}$ & $\begin{array}{c}\text { Training } \\
\text { Error Rate }\end{array}$ & $\begin{array}{c}\text { Test } \\
\text { Error Rate }\end{array}$ & $\begin{array}{c}\text { Test } \\
\text { Accuracy }\end{array}$ \\
\hline$\gamma=2^{2}$ & $3.60 \%$ & $6.70 \%$ & $93.30 \%$ & $1.60 \%$ & $2.60 \%$ & $97.40 \%$ \\
$\gamma=0$ & $0.80 \%$ & $1.95 \%$ & $98.05 \%$ & $0.60 \%$ & $1.30 \%$ & $98.70 \%$ \\
$\gamma=2^{-3}$ & $0.20 \%$ & $0.80 \%$ & $99.20 \%$ & $0.20 \%$ & $0.35 \%$ & $99.65 \%$ \\
$\gamma=2^{-6}$ & $0.60 \%$ & $1.75 \%$ & $98.25 \%$ & $0.20 \%$ & $0.95 \%$ & $99.05 \%$ \\
$\gamma=2^{-9}$ & $1.00 \%$ & $1.90 \%$ & $98.10 \%$ & $0.40 \%$ & $1.15 \%$ & $98.85 \%$ \\
\hline
\end{tabular}

The classification results were also calculated when $\gamma=2^{-9}, 2^{-6}, 2^{0}, 2^{2}$ to verify whether the IG-KELM can perform acceptably even if the kernel parameter is set badly, as shown in Table 1 and Figure 5. In general, when the value of $\gamma$ is away from the optimal value $\left(\gamma=2^{-3}\right)$, the accuracy of KELM dropped obviously. However, the IG-KELM always performed well. Especially, if $\gamma$ was set to $2^{2}$, the test accuracy rate of KELM dropped to about $93 \%$, while the IG-KELM still performed at a high level $(97.40 \%)$. The results show that the IG-KELM is insensitive to inappropriate parameter configuration, compared with KELM.

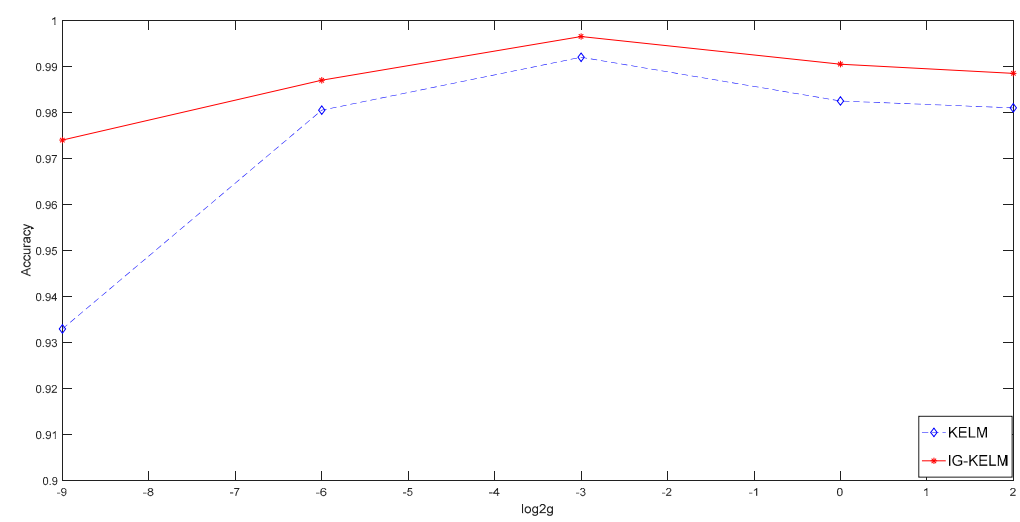

Figure 5. Comparison of test accuracy rates of KELM (blue curve) and IG-KELM (red curve) with the RBF kernel. 


\subsubsection{By Using the Polynomial Kernel}

The simulation results of KELM and IG-KELM with a Polynomial kernel were obtained when $d=2,3,4,5$, as shown in Figure 6 and Table 2.

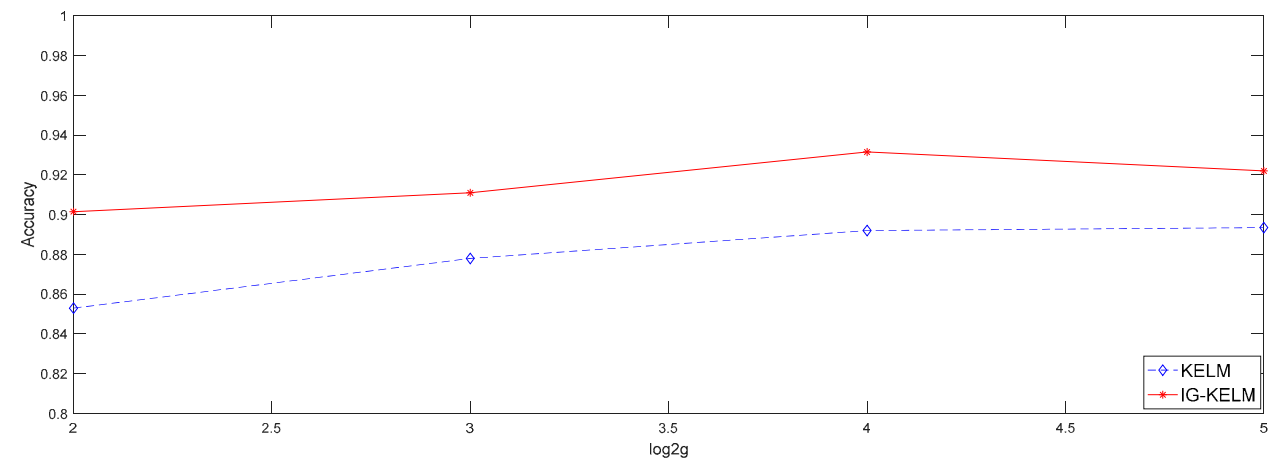

Figure 6. Comparison of test accuracy rates of KELM (blue curve) and IG-KELM (red curve) with the Polynomial kernel.

Table 2. Simulation experiments of KELM and IG-KELM with a Polynomial kernel.

\begin{tabular}{ccccccc}
\hline & \multicolumn{3}{c}{ KELM } & & IG-KELM & \\
\cline { 2 - 7 } & $\begin{array}{c}\text { Training } \\
\text { Error Rate }\end{array}$ & $\begin{array}{c}\text { Test } \\
\text { Error Rate }\end{array}$ & $\begin{array}{c}\text { Test } \\
\text { Accuracy }\end{array}$ & $\begin{array}{c}\text { Training } \\
\text { Error Rate }\end{array}$ & $\begin{array}{c}\text { Test } \\
\text { Error Rate }\end{array}$ & $\begin{array}{c}\text { Test } \\
\text { Accuracy }\end{array}$ \\
\hline$d=2$ & $9.40 \%$ & $14.70 \%$ & $85.30 \%$ & $4.20 \%$ & $9.85 \%$ & $90.15 \%$ \\
$d=3$ & $8.00 \%$ & $12.20 \%$ & $87.80 \%$ & $3.40 \%$ & $8.90 \%$ & $91.10 \%$ \\
$d=4$ & $7.80 \%$ & $10.80 \%$ & $89.20 \%$ & $2.00 \%$ & $6.85 \%$ & $93.15 \%$ \\
$d=5$ & $6.40 \%$ & $10.65 \%$ & $89.35 \%$ & $2.40 \%$ & $7.80 \%$ & $92.20 \%$ \\
\hline
\end{tabular}

Since the polynomial kernel is obviously inappropriate for the dataset, all test accuracy rates of KELM are below $90 \%$. However, the IG-KELM improves the performance rapidly even with a bad kernel type. When $d$ was set to 4 , the test accuracy rate of IG-KELM reached to $93.15 \%$. It is obvious that the IG-KELM has strong robustness to the inappropriate kernel types, compared with KELM.

\section{Application Cases of the Proposed Diagnosis Method}

\subsection{Application on Bearing Fault Diagnosis}

Rolling bearing is one of the most wildly used component in rotating machineries. Therefore, the vibration data of bearings were used to verify the propose method.

\subsubsection{Experimental Setup of Bearing}

The data from the bearing data center of Case Western Reserve University were used in this study. The test rig contains a $2 \mathrm{HP}$ motor, a torque converter/encoder, a dynamometer and control electronics, and is shown in Figure 7 (More details about the test rig can be found in http:/ /www. eecs.case.edu/laboratory/bearing/welcome_overview.htm.). The 6205-2RS JEM SKF deep-groove ball bearings were tested under four operation conditions (condition A, B, C, D), corresponding to different motor speeds and loads, as shown in Table 3. The vibration signals, including normal, inner race fault, outer race fault and rolling element fault signals were collected with a sampling rate of $12 \mathrm{kHz}$ under every condition. There are four groups of data (group A, B, C, D), corresponding to four operation conditions. Each of four groups contains 50 training samples and 100 test samples, as shown in Table 3. To verify the proposed diagnosis method under unknown variable conditions, we trained four models corresponding to four conditions respectively, and tested each of them under every condition without any prior knowledge or human intervention. 
Table 3. Experimental datasets of bearing fault diagnosis.

\begin{tabular}{ccccccccccc}
\hline \multirow{2}{*}{$\begin{array}{c}\text { Operation } \\
\text { Condition }\end{array}$} & $\begin{array}{c}\text { Motor } \\
\text { Speed }\end{array}$ & $\begin{array}{c}\text { Motor } \\
\text { Load }\end{array}$ & \multicolumn{2}{c}{ Normal } & \multicolumn{3}{c}{ Inner Race Fault } & \multicolumn{2}{c}{ Outer Race Fault } & \multicolumn{2}{c}{ Rolling Element Fault } \\
\cline { 4 - 10 } & $($ HP) & Training & Test & Training & Test & Training & Test & Training & Test \\
\hline A & 1797 & 0 & 20 & 40 & 10 & 20 & 10 & 20 & 10 & 20 \\
B & 1772 & 1 & 20 & 40 & 10 & 20 & 10 & 20 & 10 & 20 \\
C & 1750 & 2 & 20 & 40 & 10 & 20 & 10 & 20 & 10 & 20 \\
D & 1730 & 3 & 20 & 40 & 10 & 20 & 10 & 20 & 10 & 20 \\
& Total & & 80 & 160 & 40 & 80 & 40 & 80 & 40 & 80 \\
\hline
\end{tabular}

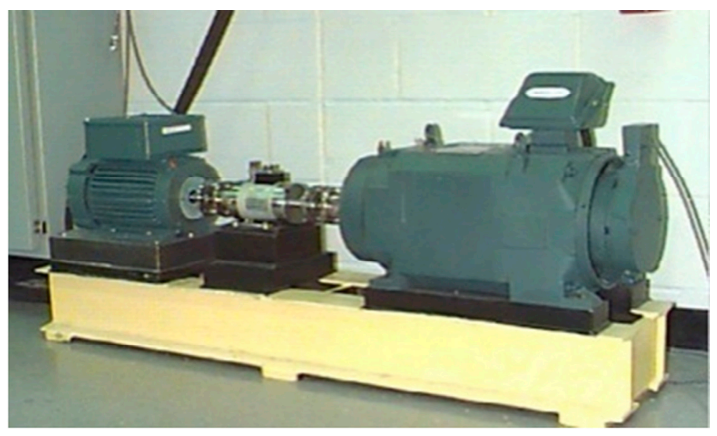

Figure 7. Experimental test rig in bearing data center.

\subsubsection{Feature Extraction Based on VMD-SVD}

Each data sample was decomposed into $n$ IMFs by VMD. In this study, $n$ was set to 8 by default. Take a normal signal for example, the processing result is shown in Figure 8. For comparison, the results by EMD was also obtained, as shown in Figure 9. Compared with results of VMD and EMD, it is shown that VMD can separate signals more effectively and eliminate the modal aliasing problem.

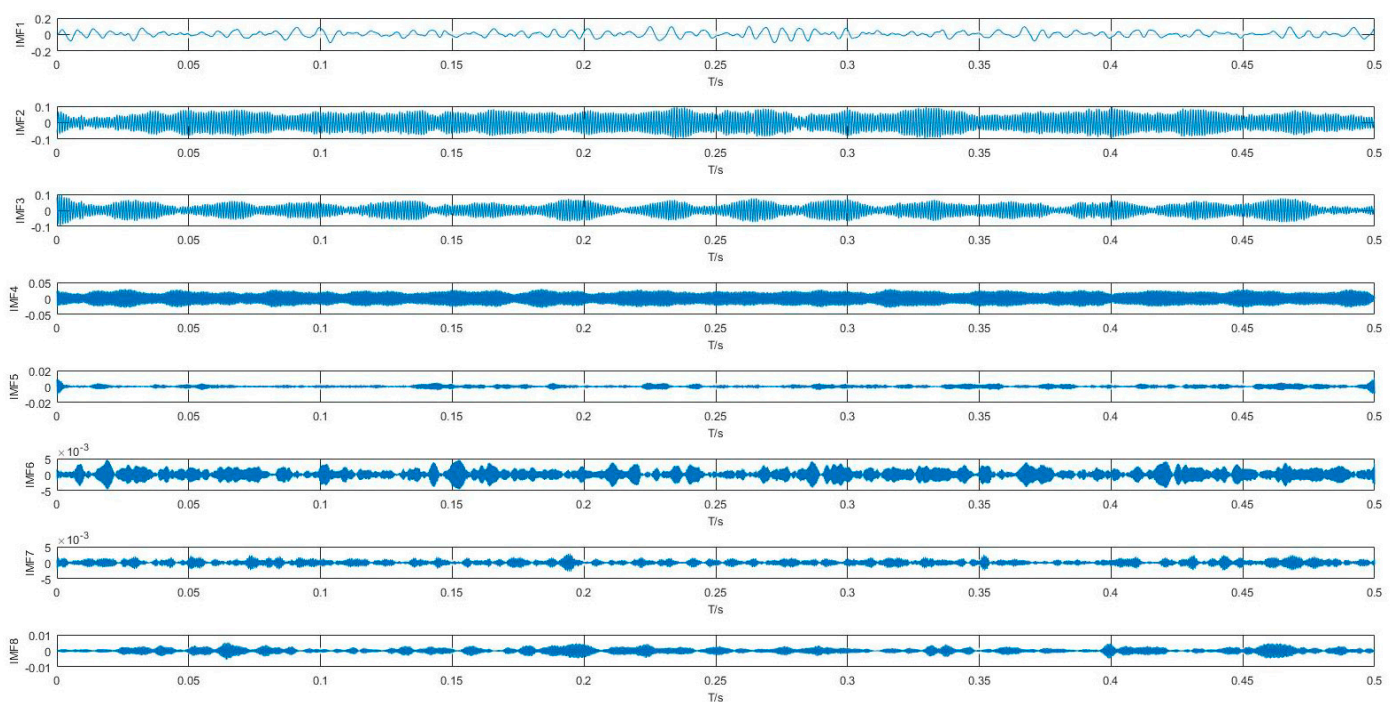

Figure 8. IMFs obtained by VMD from the normal signal. 

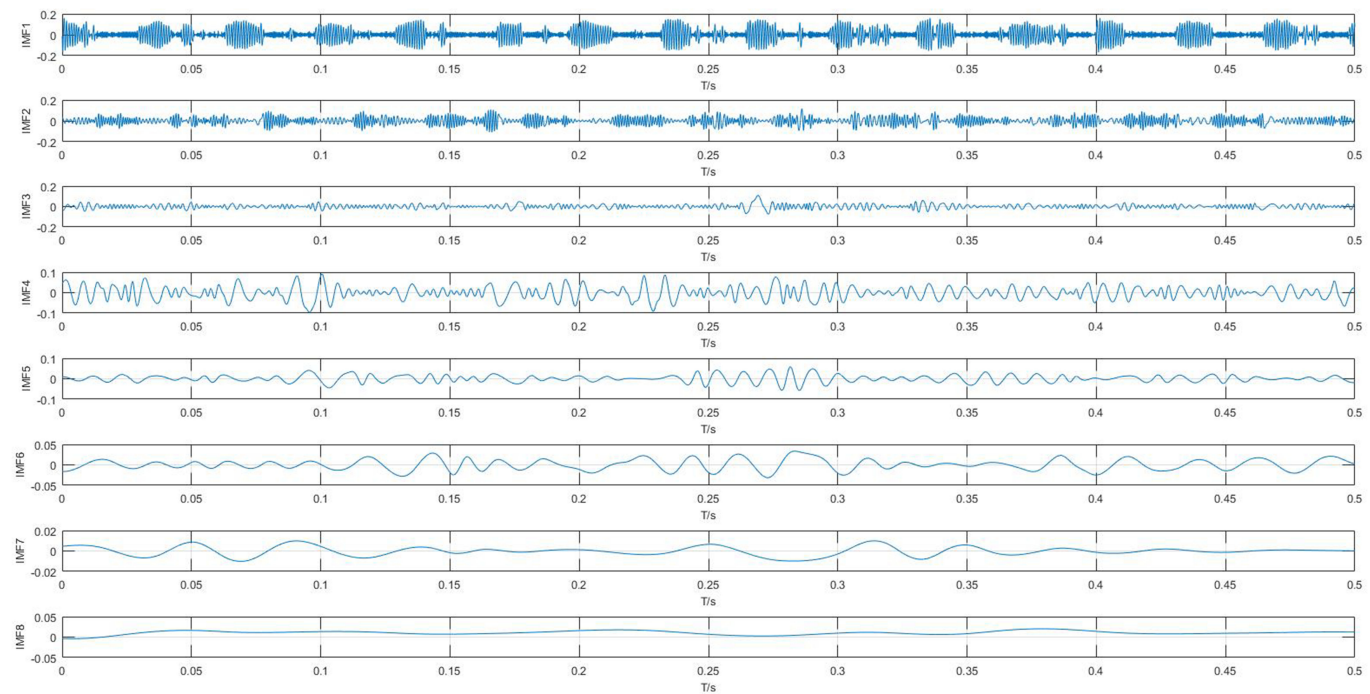

Figure 9. IMFs obtained by EMD from the normal signal.

The IMFs were used to construct the matrix. Then, the singular values can be acquired by using SVD. Take Condition A for example, the results are shown in Figure 10. Compared with results of EMD-SVD (as shown in Figure 11), for each certain fault mode, the results obtained by VMD-SVD are more coincident and stable, respectively. Different fault modes can be easily distinguishable by using VMD-SVD. On the contrary, the results of EMD-SVD show more variability. That is obviously a disadvantage to fault diagnosis.
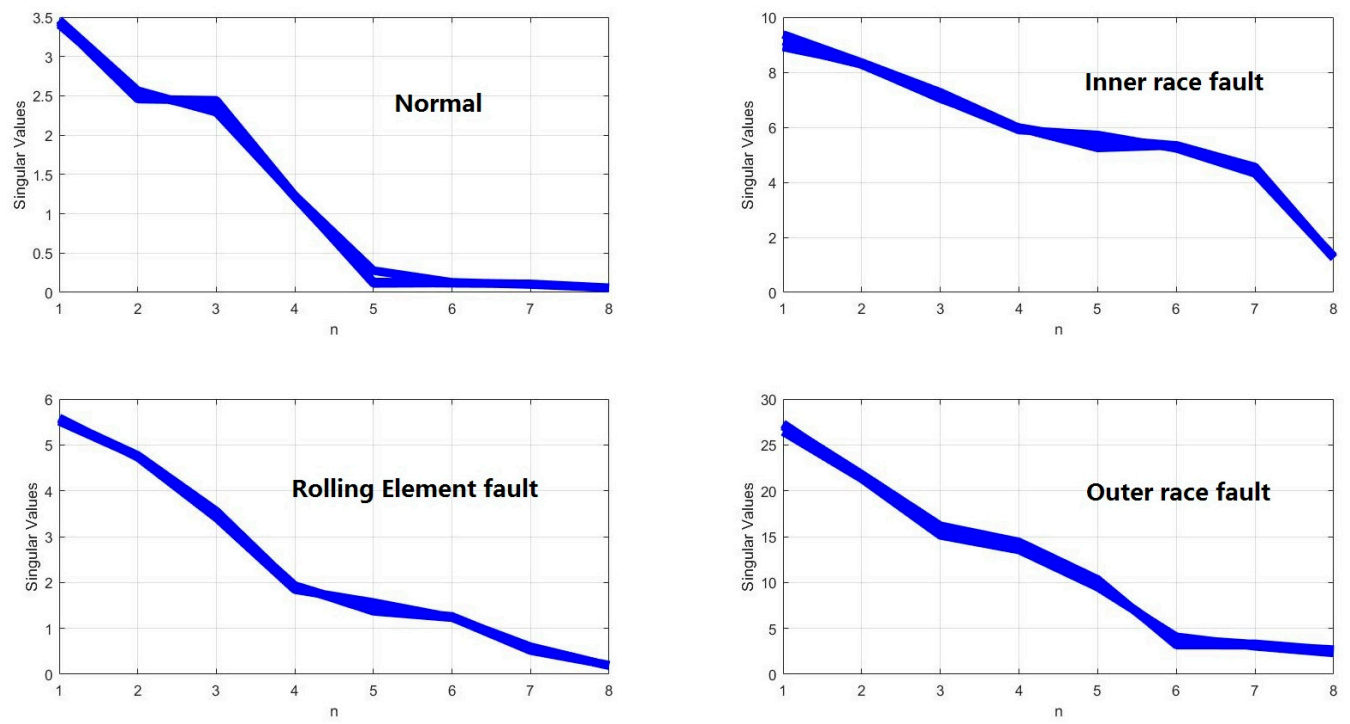

Figure 10. Singular values obtained by VMD-SVD. 

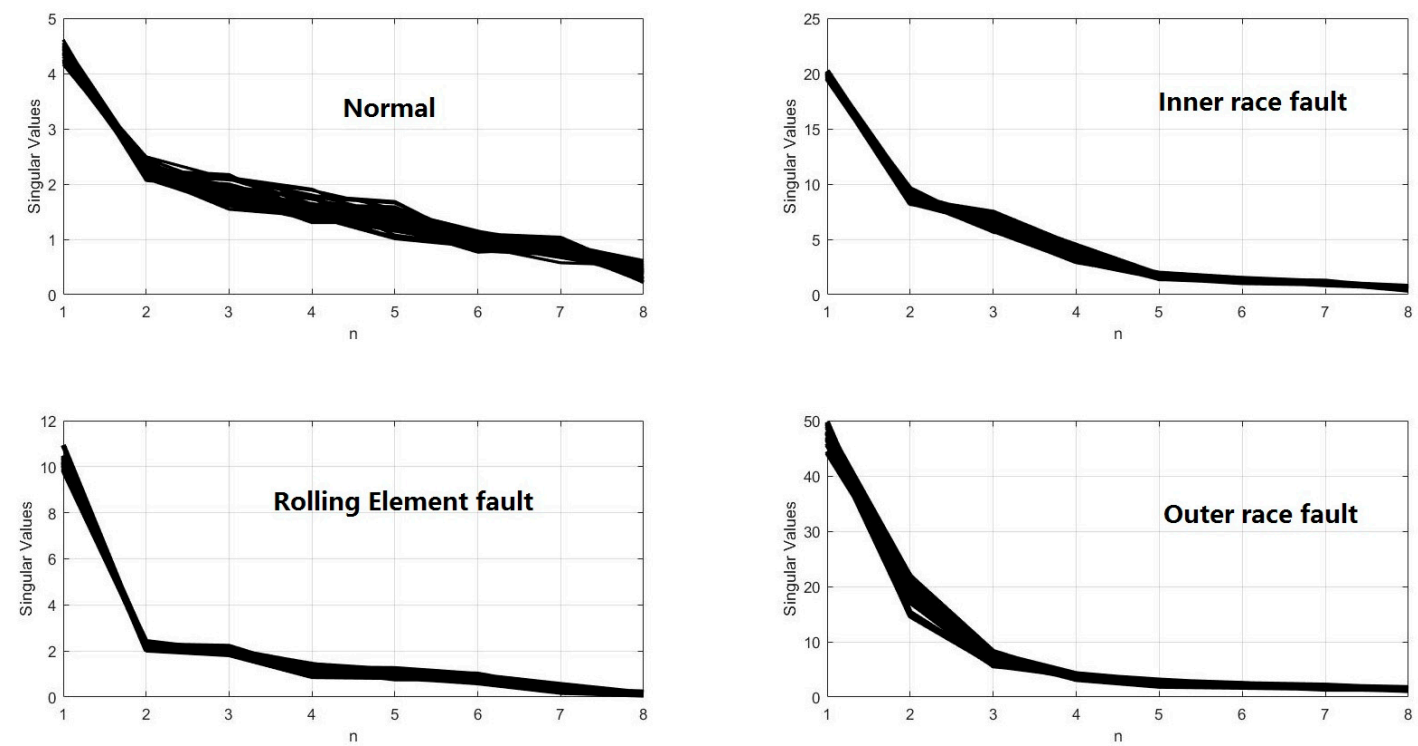

Figure 11. Singular values obtained by EMD-SVD.

\subsubsection{Fault Clustering for Bearing}

In this study, IG-KELM with an RBF kernel was employed for fault clustering based on features extracted by VMD-SVD. Four trained models, corresponding to four conditions were obtained and tested under every operation condition, respectively, as shown in Table 4. The conventional KELM was employed for comparison.

In this study, all training errors of KELM and IG-KELM models were calculated to be zero; if the trained model and test samples come from the same condition, all test accuracy rates are $100 \%$ (which means the test errors are zero). That is mainly due to the efficiency of the hybrid feature extraction method (VMD-SVD). However, if the trained model and test samples come from different conditions, the test accuracy rates of KELM decrease rapidly. When the trained KELM from Condition B was employed under Condition D, the test accuracy rate was only $89.25 \%$, which is unacceptable in applications.

Table 4. Results of bearing fault diagnosis.

\begin{tabular}{cccccc}
\hline \multirow{3}{*}{ Operation Condition } & \multirow{3}{*}{ Model } & \multicolumn{4}{c}{ Operation Condition } \\
\cline { 3 - 6 } & & A & B & C & D \\
\cline { 3 - 6 } & & Accuracy & Accuracy & Accuracy & Accuracy \\
\hline \multirow{2}{*}{$\mathrm{A}$} & KELM & $100 \%$ & $99.50 \%$ & $99.25 \%$ & $99.25 \%$ \\
& IG-KELM & $100 \%$ & $100 \%$ & $100 \%$ & $100 \%$ \\
\multirow{2}{*}{$\mathrm{B}$} & KELM & $94.75 \%$ & $100 \%$ & $99.75 \%$ & $89.25 \%$ \\
& IG-KELM & $96.50 \%$ & $100 \%$ & $100 \%$ & $96.50 \%$ \\
C & KELM & $93.00 \%$ & $98.50 \%$ & $100 \%$ & $91.50 \%$ \\
& IG-KELM & $97.75 \%$ & $99.50 \%$ & $100 \%$ & $98.25 \%$ \\
D & KELM & $96.50 \%$ & $97.75 \%$ & $92.75 \%$ & $100 \%$ \\
& IG-KELM & $97.75 \%$ & $100 \%$ & $98.50 \%$ & $100 \%$ \\
\hline
\end{tabular}

On the contrary, IG-KELM performed much better under unknown variable conditions and the test accuracy rates were no less than $96 \%$. Compared with the strong sensitivity of KELM to the operation condition, the IG-KELM is able to adapt itself in a data-dependent way and improves the performance under unknown variable conditions rapidly. The IG-KELM can be implemented even without any prior knowledge about the current operation condition.

Therefore, the novel method using IG-KELM based on VMD-SVD can be utilized for bearing fault diagnosis under unknown variable conditions with little prior knowledge or human intervention. 


\subsection{Application on Hydraulic Pump Fault Diagnosis}

The proposed method was also applied on fault diagnosis of a hydraulic pump. The vibration signals were gathered from a test rig of SCY hydraulic plunger pump with a sampling rate of $1000 \mathrm{~Hz}$, as shown in Figure 12. The nominal pressure is $31.5 \mathrm{Mpa}$, and the nominal displacement is $1.25-400 \mathrm{~mL} / \mathrm{r}$. The pump was running at a fluctuant motor speed of $5280 \pm 200 \mathrm{rpm}$ while gathering signals. Vibration signals were collected using a four-channel DAT recorder. Considering that the most crucial fault modes of the plunger pump are slipper loosing and valve plate wear, the dataset consists of three types of states, corresponding to no trouble (Normal), slipper loosing (Fault 1 ) and valve plate wear (Fault 2), as shown in Table 5.

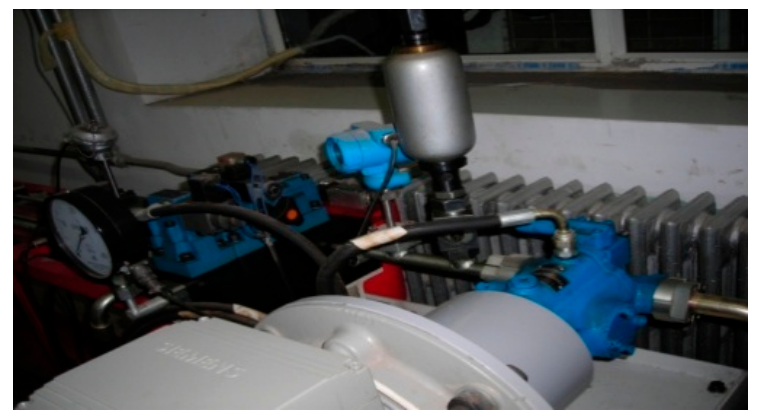

Figure 12. Experimental test rig of SCY hydraulic plunger pump.

Table 5. Experimental datasets of hydraulic pump fault diagnosis.

\begin{tabular}{ccccc}
\hline \multirow{2}{*}{ Type } & \multicolumn{3}{c}{ SCY Plunger Pump } & \multirow{2}{*}{ Total } \\
\cline { 2 - 4 } & Normal & Slipper Loosing & Valve Plate Wear & \\
\hline No. of training samples & 30 & 30 & 30 & 90 \\
No. of test samples & 30 & 30 & 30 & 90 \\
\hline
\end{tabular}

The proposed method was applied on the dataset and KELM was also used for comparison, as shown in Table 6, from which we can see that KELM has a high false alarm rate (25/30). That is because some normal data under unknown fluctuant conditions were identified as abnormal data by the KELM model. On the contrary, the IG-KELM has strong robustness to fluctuant conditions and can reduce the false alarms and improve the classification accuracy. The results show that the application of the proposed method on hydraulic pump fault diagnosis is feasible and efficient.

Table 6. Results of hydraulic pump fault diagnosis.

\begin{tabular}{ccccccc}
\hline \multirow{3}{*}{ Model } & \multicolumn{3}{c}{ KELM } & \multicolumn{3}{c}{ IG-KELM } \\
\cline { 2 - 7 } & $\begin{array}{c}\text { Training } \\
\text { Error }\end{array}$ & $\begin{array}{c}\text { Test } \\
\text { Error }\end{array}$ & $\begin{array}{c}\text { Test } \\
\text { Accuracy }\end{array}$ & $\begin{array}{c}\text { Training } \\
\text { Error }\end{array}$ & $\begin{array}{c}\text { Test } \\
\text { Error }\end{array}$ & $\begin{array}{c}\text { Test } \\
\text { Accuracy }\end{array}$ \\
\hline Normal & $3 / 30$ & $5 / 30$ & $25 / 30$ & $0 / 30$ & $0 / 30$ & $30 / 30$ \\
Slipper loosing & $0 / 30$ & $1 / 30$ & $29 / 30$ & $0 / 30$ & $0 / 30$ & $30 / 30$ \\
Valve plate wear & $1 / 30$ & $3 / 30$ & $27 / 30$ & $0 / 30$ & $2 / 30$ & $28 / 30$ \\
Total & $4 / 90$ & $9 / 90$ & $81 / 90$ & $0 / 90$ & $2 / 90$ & $88 / 90$ \\
Percentage & $4.44 \%$ & $10.00 \%$ & $90.00 \%$ & $100 \%$ & $2.22 \%$ & $97.78 \%$ \\
\hline
\end{tabular}

\section{Conclusions}

This paper focuses on adaptive diagnosis for rotating machineries, which can diagnose faults automatically under unknown variable operation conditions with little prior knowledge or human intervention. For this end, this paper proposes a method using IG-KELM based on VMD-SVD for fault diagnosis. Firstly, the VMD-SVD method is employed to extract features from the vibration 
signals self-adaptively. Secondly, IG-KELM, which employs information geometry to modify KELM data-dependently is used for fault clustering. The IG-KELM can be modified self-adaptively, and be insensitive to the manual configuration of kernel. The simulation results show that IG-KELM can increase the accuracy rate by up to $4.85 \%$. Therefore, IG-KELM can perform efficiently even if the kernel types or parameters are set badly. Finally, the proposed method was applied on fault diagnosis of bearing and hydraulic pump under variable conditions. Compared with the conventional method, the proposed method increased the accuracy rates by up to $7.25 \%$ and $7.78 \%$, respectively. The results show that the proposed method has strong self-adaptivity and high accuracy during feature extraction and fault clustering.

Considering that this proposed method can be applied automatically and require little prior knowledge or human intervention, it can be available on digital signal processors (DSPs), field programmable gate arrays (FPGAs) or even smartphones.

However, to some extent, the operation conditions involved in this study only fluctuated in a narrow region. Larger differences of operation conditions may create new problems. Therefore, additional experiments under more different conditions should be done to validate and improve the method. Meanwhile, more attentions should be paid to the development of more and better adaptive diagnosis methods.

Acknowledgments: This research is supported by the National Key R\&D Program of China (No. 2016YFB1200203), the State Key Laboratory of Rail Traffic Control and Safety (Contract Nos. RCS2016ZQ003 and RCS2016ZT018), Beijing Jiaotong University, as well as National Engineering Laboratory for System Safety and Operation Assurance of Urban Rail Transit.

Author Contributions: L.J. and Y.Q. conceived and designed the experiments; Y.Q. performed the experiments; L.J. and Z.W. analyzed the data; Z.W. wrote the paper.

Conflicts of Interest: The authors declare no conflict of interest.

\section{References}

1. Azadeh, A.; Saberi, M.; Kazem, A.; Ebrahimipour, V.; Nourmohammadzadeh, A.; Saberi, Z. A flexible algorithm for fault diagnosis in a centrifugal pump with corrupted data and noise based on ANN and support vector machine with hyper-parameters optimization. Appl. Soft Comput. 2013, 13, 1478-1485. [CrossRef]

2. Li, B.; Liu, P.Y.; Hu, R.X.; Mi, S.S.; Fu, J.P. Fuzzy lattice classifier and its application to bearing fault diagnosis. Appl. Soft Comput. 2012, 12, 1708-1719. [CrossRef]

3. Grebenik, J.; Zhang, Y.; Bingham, C.; Srivastava, S. Roller element bearing acoustic fault detection using smartphone and consumer microphones comparing with vibration techniques. In Proceedings of the 17th International Conference on Mechatronics-Mechatronika (ME), Prague, Czech Republic, 7-9 December 2016; pp. 1-7.

4. Rzeszucinski, P.; Orman, M.; Pinto, C.T.; Tkaczyk, A.; Sulowicz, M. A signal processing approach to bearing fault detection with the use of a mobile phone. In Proceedings of the 2015 IEEE 10th International Symposium on Diagnostics for Electrical Machines, Power Electronics and Drives (SDEMPED), Guarda, Portugal, 1-4 September 2015; pp. 310-315.

5. Belfiore, N.P.; Rudas, I.J. Applications of computational intelligence to mechanical engineering. In Proceedings of the 2014 IEEE 15th International Symposium on Computational Intelligence and Informatics (CINTI), Budapest, Hungary, 19-21 November 2014; pp. 351-368.

6. Wanbin, W.; Tse, P.W. Remote Machine Monitoring Through Mobile Phone, Smartphone or PDA. In Proceedings of the 1st World Congress on Engineering Asset Management (WCEAM), Gold Coast, Australia, 11-14 July 2006; pp. 309-315.

7. Shields, D.N.; Damy, S. A quantitative fault detection method for a class of nonlinear systems. Trans. Inst. Meas. Control 1998, 20, 125-133. [CrossRef]

8. Sakthivel, N.R.; Sugumaran, V.; Nair, B.B. Automatic rule learning using roughset for fuzzy classifier in fault categorization of mono-block centrifugal pump. Appl. Soft Comput. 2012, 12, 196-203. [CrossRef] 
9. Shengqiang, W.; Yuru, M.; Wanlu, J.; Sheng, Z. Kernel principal component analysis fault diagnosis method based on sound signal processing and its application in hydraulic pump. In Proceedings of the 2011 International Conference on Fluid Power and Mechatronics, Beijing, China, 17-20 August 2011; pp. 98-101.

10. Yu, J.B. A hybrid feature selection scheme and self-organizing map model for machine health assessment. Appl. Soft Comput. 2011, 11, 4041-4054. [CrossRef]

11. Liu, H.; Wang, X.; Lu, C. Rolling bearing fault diagnosis based on LCD-TEO and multifractal detrended fluctuation analysis. Mech. Syst. Signal Process. 2015, 60-61, 273-288. [CrossRef]

12. Tian, Y.; Wang, Z.; Lu, C. Self-adaptive bearing fault diagnosis based on permutation entropy and manifold-based dynamic time warping. Mech. Syst. Signal Process. 2016. [CrossRef]

13. Tian, Y.; Ma, J.; Lu, C.; Wang, Z. Rolling bearing fault diagnosis under variable conditions using LMD-SVD and extreme learning machine. Mech. Mach. Theory 2015, 90, 175-186. [CrossRef]

14. Wu, J.D.; Huang, C.K. An engine fault diagnosis system using intake manifold pressure signal and Wigner-Ville distribution technique. Expert Syst. Appl. 2011, 38, 536-544. [CrossRef]

15. Baydar, N.; Ball, A. A comparative study of acoustic and vibration signals in detection of gear failures using wigner-ville distribution. Mech. Syst. Signal Process. 2001, 15, 1091-1107. [CrossRef]

16. Jing, L.; Zhao, M.; Li, P.; Xu, X. A convolutional neural network based feature learning and fault diagnosis method for the condition monitoring of gearbox. Measurement 2017, 111, 1-10. [CrossRef]

17. Li, B.; Zhang, P.L.; Tian, H.; Mi, S.S.; Liu, D.S.; Ren, G.Q. A new feature extraction and selection scheme for hybrid fault diagnosis of gearbox. Expert Syst. Appl. 2011, 38, 10000-10009. [CrossRef]

18. Lou, X.; Loparo, K.A. Bearing fault diagnosis based on wavelet transform and fuzzy inference. Mech. Syst. Signal Process. 2004, 18, 1077-1095. [CrossRef]

19. Wu, J.D.; Chen, J.C. Continuous wavelet transform technique for fault signal diagnosis of internal combustion engines. NDT \& E Int. 2006, 39, 304-311.

20. Su, W.; Wang, F.; Zhu, H.; Zhang, Z.; Guo, Z. Rolling element bearing faults diagnosis based on optimal Morlet wavelet filter and autocorrelation enhancement. Mech. Syst.Signal Process. 2010, 24, 1458-1472. [CrossRef]

21. Zou, J.; Chen, J. A comparative study on time-frequency feature of cracked rotor by Wigner-Ville distribution and wavelet transform. J. Sound Vib. 2004, 276, 1-11. [CrossRef]

22. Yan, R.Q.; Gao, R.X. An efficient approach to machine health diagnosis based on harmonic wavelet packet transform. Robot Comput. Manuf. 2005, 21, 291-301. [CrossRef]

23. Tse, P.W.; Yang, W.X.; Tam, H.Y. Machine fault diagnosis through an effective exact wavelet analysis. J. Sound Vib. 2004, 277, 1005-1024. [CrossRef]

24. Rafiee, J.; Rafiee, M.A.; Tse, P.W. Application of mother wavelet functions for automatic gear and bearing fault diagnosis. Expert Syst. Appl. 2010, 37, 4568-4579. [CrossRef]

25. Rafiee, J.; Tse, P.W.; Harifi, A.; Sadeghi, M.H. A novel technique for selecting mother wavelet function using an intelligent fault diagnosis system. Expert Syst. Appl. 2009, 36, 4862-4875. [CrossRef]

26. Junsheng, C.; Dejie, Y.; Yu, Y. Research on the intrinsic mode function (IMF) criterion in EMD method. Mech. Syst. Signal Process. 2006, 20, 817-824. [CrossRef]

27. Huang, N.E.; Wu, M.L.; Qu, W.; Long, S.R.; Shen, S.S.P. Applications of Hilbert-Huang transform to non-stationary financial time series analysis. Appl. Stoch. Models Bus. Ind. 2003, 19, 245-268. [CrossRef]

28. Huang, N.E.; Shen, Z.; Long, S.R.; Wu, M.C.; Shih, H.H.; Zheng, Q.; Yen, N.C.; Tung, C.C.; Liu, H.H. The Empirical Mode Decomposition and the Hilbert Spectrum for Nonlinear and Non-Stationary Time Series Analysis. Proc. Math. Phys. Eng. Sci. 1998, 454, 903-995. [CrossRef]

29. Yu, D.; Cheng, J.; Yang, Y. Application of EMD method and Hilbert spectrum to the fault diagnosis of roller bearings. Mech. Syst. Signal Process. 2005, 19, 259-270. [CrossRef]

30. Junsheng, C.; Dejie, Y.; Yu, Y. A fault diagnosis approach for roller bearings based on EMD method and AR model. Mech. Syst. Signal Process. 2006, 20, 350-362. [CrossRef]

31. Cheng, G.; Cheng, Y.L.; Shen, L.H.; Qiu, J.B.; Zhang, S. Gear fault identification based on Hilbert-Huang transform and SOM neural network. Measurement 2013, 46, 1137-1146. [CrossRef]

32. Rai, V.K.; Mohanty, A.R. Bearing fault diagnosis using FFT of intrinsic mode functions in Hilbert-Huang transform. Mech.Syst. Signal Process. 2007, 21, 2607-2615. [CrossRef]

33. Dragomiretskiy, K.; Zosso, D. Variational mode decomposition. IEEE Trans. Signal Process. 2014, 62, 531-544. [CrossRef] 
34. Wang, Y.; Markert, R.; Xiang, J.; Zheng, W. Research on variational mode decomposition and its application in detecting rub-impact fault of the rotor system. Mech. Syst. Signal Process. 2015, 60-61, 243-251. [CrossRef]

35. Cheng, J.S.; Yu, D.J.; Tang, J.S.; Yang, Y. Application of SVM and SVD technique based on EMD to the fault diagnosis of the rotating machinery. Shock Vib. 2009, 16, 89-98. [CrossRef]

36. Veen, A.J.V.D.; Deprettere, E.F.; Swindlehurst, A.L. Subspace-based signal analysis using singular value decomposition. Proc. IEEE 1993, 81, 1277-1308. [CrossRef]

37. Lin, J.; Chen, Q. Fault diagnosis of rolling bearings based on multifractal detrended fluctuation analysis and Mahalanobis distance criterion. Mech. Syst. Signal Process. 2013, 38, 515-533. [CrossRef]

38. Wang, P.C.; Su, C.T.; Chen, K.H.; Chen, N.H. The application of rough set and Mahalanobis distance to enhance the quality of OSA diagnosis. Expert Syst. Appl. 2011, 38, 7828-7836. [CrossRef]

39. Wang, Z.; Lu, C.; Wang, Z.; Liu, H.; Fan, H. Fault diagnosis and health assessment for bearings using the Mahalanobis-Taguchi system based on EMD-SVD. Trans. Inst. Meas. Control 2013, 35, 798-807. [CrossRef]

40. Li, J.; Cui, P. Improved kernel fisher discriminant analysis for fault diagnosis. Expert Syst. Appl. 2009, 36, 1423-1432. [CrossRef]

41. Md Nor, N.; Hussain, M.A.; Che Hassan, C.R. Fault diagnosis and classification framework using multi-scale classification based on kernel Fisher discriminant analysis for chemical process system. Appl. Soft Comput. 2017, 61, 959-972. [CrossRef]

42. Ekici, S. Support Vector Machines for classification and locating faults on transmission lines. Appl. Soft Comput. 2012, 12, 1650-1658. [CrossRef]

43. Muralidharan, V.; Sugumaran, V. A comparative study of naive bayes classifier and Bayes net classifier for fault diagnosis of monoblock centrifugal pump using wavelet analysis. Appl. Soft Comput. 2012, 12, 2023-2029. [CrossRef]

44. Jin, C.; Jin, S.W.; Qin, L.N. Attribute selection method based on a hybrid BPNN and PSO algorithms. Appl. Soft Comput. 2012, 12, 2147-2155. [CrossRef]

45. Koupaei, J.A.; Abdechiri, M. Sensor deployment for fault diagnosis using a new discrete optimization algorithm. Appl. Soft Comput. 2013, 13, 2896-2905. [CrossRef]

46. Sanz, J.; Perera, R.; Huerta, C. Gear dynamics monitoring using discrete wavelet transformation and multi-layer perceptron neural networks. Appl. Soft Comput. 2012, 12, 2867-2878. [CrossRef]

47. Juntunen, P.; Liukkonen, M.; Lehtola, M.; Hiltunen, Y. Cluster analysis by self-organizing maps: An application to the modelling of water quality in a treatment process. Appl. Soft Comput. 2013, 13, 3191-3196. [CrossRef]

48. Lu, C.; Ma, N.; Wang, Z.P. Fault detection for hydraulic pump based on chaotic parallel RBF network. EURASIP J. Adv. Signal Process. 2011, 1, 49. [CrossRef]

49. Jia, F.; Lei, Y.G.; Lin, J.; Zhou, X.; Lu, N. Deep neural networks: A promising tool for fault characteristic mining and intelligent diagnosis of rotating machinery with massive data. Mech. Syst. Signal Process. 2016, 72-73, 303-315. [CrossRef]

50. Tran, V.T.; AlThobiani, F.; Ball, A. An approach to fault diagnosis of reciprocating compressor valves using Teager-Kaiser energy operator and deep belief networks. Expert Syst. Appl. 2014, 41, 4113-4122. [CrossRef]

51. Shao, H.; Jiang, H.; Lin, Y.; Li, X. A novel method for intelligent fault diagnosis of rolling bearings using ensemble deep auto-encoders. Mech. Syst. Signal Process. 2018, 102, 278-297. [CrossRef]

52. Huang, G.B.; Zhu, Q.Y.; Siew, C.K. Extreme learning machine: Theory and applications. Neurocomputing 2006, 70, 489-501. [CrossRef]

53. Huang, G.B.; Zhu, Q.Y.; Siew, C.K. Extreme learning machine: A new learning scheme of feedforward neural networks. In Proceedings of the 2004 IEEE International Joint Conference on Neural Networks, Budapest, Hungary, 25-29 July 2004; pp. 985-990.

54. Huang, G.; Huang, G.B.; Song, S.; You, K. Trends in extreme learning machines: A review. Neural Netw. 2015, 61, 32-48. [CrossRef] [PubMed]

55. Wan, Y.; Song, S.; Huang, G.; Li, S. Twin extreme learning machines for pattern classification. Neurocomputing 2017, 260, 235-244. [CrossRef]

56. Huang, G.B.; Siew, C.K. Extreme learning machine with randomly assigned RBF kernels. Int. J. Inf. Technol. 2005, 11, 16-24.

57. Rodriguez, N.; Cabrera, G.; Lagos, C.; Cabrera, E. Stationary wavelet singular entropy and kernel extreme learning for bearing multi-fault diagnosis. Entropy 2017, 19, 541. [CrossRef] 
58. Amari, S.I. Information geometry and its applications: Convex function and dually flat manifold. Emerg. Trends Vis. Comput. 2009, 5416, 75-102.

59. Amari, S.I. Information geometry in optimization, machine learning and statistical inference. Front. Electr. Electron. Eng. 2010, 5, 241-260. [CrossRef]

60. Amari, S.I. Dualistic geometry of the manifold of higher-order neurons. Neural Netw. 1991, 4, $443-451$. [CrossRef]

61. Amari, S.I. Information geometry of the EM and em algorithms for neural networks. Neural Netw. 1995, 8, 1379-1408. [CrossRef]

62. Amari, S.I. Information geometry of multilayer perceptron. Int. Congr. Ser. 2004, 1269, 3-5. [CrossRef]

63. Fujiwara, A.; Amari, S.I. Gradient systems in view of information geometry. Phys. D Nonlinear Phenomena 1995, 80, 317-327. [CrossRef]

64. Ohara, A.; Suda, N.; Amari, S.I. Dualistic differential geometry of positive definite matrices and its applications to related problems. Linear Algebra Appl. 1996, 247, 31-53. [CrossRef]

65. Wu, S.; Amari, S.I. Conformal transformation of kernel functions: A data-dependent way to improve support vector machine classifiers. Neural Process. Lett. 2002, 15, 59-67. [CrossRef]

(C) 2018 by the authors. Licensee MDPI, Basel, Switzerland. This article is an open access article distributed under the terms and conditions of the Creative Commons Attribution (CC BY) license (http://creativecommons.org/licenses/by/4.0/). 